\title{
Design, Development and Evaluation of Matrix Tablet
}

\author{
Dr. Arindam Chatterjee, Mrs. Deeksha Sharma, Anuj Mishra \\ ${ }^{1}$ Associate Professor, Department of Pharmaceutics, Jaipur College of Pharmacy, Jaipur, India \\ ${ }^{2}$ Assistant Professor, Department of Pharmaceutics, Jaipur College of Pharmacy, Jaipur, India \\ ${ }^{3}$ M.Pharm., Research Scholar, Department of Pharmaceutics, Jaipur College of Pharmacy, \\ Jaipur, India
}

\begin{abstract}
Article Info: Received 15 September 2021; Accepted 29 October 2021
DOI: https://doi.org/10.32553/jbpr.v10i6.889

Corresponding author: Anuj Mishra

Conflict of interest statement: No conflict of interest
\end{abstract}

\begin{abstract}
Recently, sustained release formulations have become a very helpful tool in medical practice, providing patients with a variety of benefits. Sustained release is also a potential method for reducing pharmacological side effects by preventing fluctuations in the concentration of the drug in the plasma. Nowadays, relatively few pharmaceuticals are emerging from research and development, and current drugs are suffering from resistance as a result of their inappropriate usage. Thus, altering the operation is an appropriate and optimum method of increasing the effectiveness of a medicine by a little variation in the drug distribution. The release of the medication through such a system is regulated by both dissolution and diffusion processes. Most medications, if not correctly designed, may quickly release the drug at a higher pace, resulting in hazardous concentrations of the drug upon oral administration. This review discusses the fundamentals of sustained release formulations and the many varieties available.
\end{abstract}

Key words: Matrix tablets, Sustained release, Sustain release polymers, Patient convenience and compliance.

\section{Introduction}

The oral route is the most often utilised route for drug administration, owing to its simplicity of administration and the fact that gastrointestinal physiology allows for more design freedom than most other routes ${ }^{1}$. Sustained release, prolonged release, modified release, extended release, and depot formulations are all terms that refer to drug delivery systems that are intended to achieve or extend therapeutic effect by continuously releasing medication over an extended period of time following administration of a single dose ${ }^{2}$. For some years, the Pharmaceutical industry has recognised the benefits of providing a single dosage of a medicine that is delivered over a long period of time rather than several doses. The goal to keep a drug's blood level near constant or uniform often translates into improved patient compliance and increased clinical effectiveness for the drug's intended use ${ }^{3}$. Due to the increasing complexity and cost associated with selling novel pharmacological entities, has shifted its emphasis to developing sustained or controlled release drug delivery systems ${ }^{4}$. The matrix system is commonly employed in the pharmaceutical industry for the aim of sustained 
release. It is the release mechanism that prolongs and regulates the release of dissolved or dispersed drugs. Indeed, a matrix is defined as a homogeneous mixture of one or more medicines and a gelling agent, i.e. hydrophilic polymers ${ }^{5}$. Prolonged release dosage forms are intended to sustain therapeutic medication levels in the plasma for an extended length of time.

\section{The principal disadvantages of conventional dosage forms are as follows: ${ }^{6}$}

- Patient noncompliance.

- Increased likelihood of skipping a dosage of a medicine with a short half-life that requires frequent administration.

- Unavoidable changes in drug concentrations might result in under- or over-medication.

- A characteristic peak-valley plasma concentration time curve is produced, making steady-state conditions challenging to achieve.

Due to the growing complexity and cost associated with launching novel pharmacological entities, has shifted its emphasis to the development of sustained or controlled release drug delivery systems ${ }^{7}$.

Matrix methods are often employed to provide continuous release. It is the release mechanism that prolongs and regulates the release of dissolved or dispersed drugs. Due to its simplicity of administration, patient compliance, lack of sterility requirements, and adaptability of dose forms, the oral route has been one of the most common means of drug delivery. Time release technology, alternatively referred to as sustained release (SR), sustained action (SA), extended release (ER, XR, or XL), time-release or timed-release, controlled-release (CR), modified release (MR), or continuous-release (CR), is a mechanism used in pill tablets or capsules to dissolve slowly and release a drug over an extended period of time. The matrix system is commonly employed in the pharmaceutical industry for the aim of sustained release. It is the mechanism that prolongs and regulates the release of dissolved or distributed drugs.

\section{Matrix Tablets}

The most popular controlled release drug delivery device is the matrix tablet, which releases the medication by diffusion or a controlled dissolving process. The active ingredient is equally spread throughout the ratelimiting substance, which may be hydrophilic, plastic, lipid, or mineral based. The polymeric material works as an inhibitor to the rate of release. Thus, it maintains a consistent therapeutic level in the blood and avoids fluctuations, i.e., the minimal or toxic concentration, thus preventing local or systemic adverse responses. Different kinds of matrices exhibit distinct release patterns, and hence the distinct features of matrix substances aid in indicating the drug release pattern.

\section{Advantages of oral controlled release matrix tablets}

1. Reduction in dosing frequency.

2. Reduction in plasma drug concentration.

3. Better management of therapy.

4. Improve Patients compliance.

5. Therapeutic Advantage.

6. Reduction in Adverse Effects.

7. Cost Effective.

8. Drug stability enhance by shielding the active ingredient from hydrolysis and degradation.

9. Release high molecular weight compound effectively.

\section{Disadvantages of oral controlled release matrix tablets}

1. Decreased systemic availability as compare to conventional tablets because it is used to enhanced first-pass effects, more instability, incomplete release, inadequate gastric residence, particular site absorption, $\mathrm{pH}$ dependent stability.

2. The release rate of drugs can also be changed by meal and gastric emptying time.

3. Break down of tablet lose controlled release property.

4. Development cost increase due to specialized equipment and expensive excipients. 
5. In-vitro in-vivo correlation (IVIVC) required thorough analysis.

6. Dose adjustment of drugs given in different strength becomes difficult ${ }^{7}$.

\section{Methods for Preparation of matrix tablet}

\section{Direct compression}

Powders or granules are compressed directly to form tablets without changing the physical property ${ }^{8}$.

\section{Dry granulation}

Dry granulation is of two types: slugging and roller compaction. In slugging method, granule is re-compressed and slugs are crushed to produce granules. Whereas in roller compaction, powder is re-compress with pressure rolls.

\section{Wet granulation}

It involves massing of dry granule blends in a volatile fluid, then wet sizing is done, after that drying is done and it is followed by dry screening.

\section{Steam granulation}

Steam is used as a binder for granulation rather than water. It uniformly distributes and diffuses into the granules. The granules become rounded having more surface area and hence it enhance drug dissolution rate from granules ${ }^{9}$.

\section{Melt granulation}

Moldable binders are used for granulation, which melts at $50-80{ }^{\circ} \mathrm{C}$. Dry granules are obtained by cooling it at ambient temperature.

\section{Freeze granulation}

It involves the spraying of droplets in slurry into liquid nitrogen and the drops are then immediately frozen into granules followed by drying process, i.e. lyophilisation.

\section{Foam granulation}

Aqueous binders are added as foam agent which increases surface area of foam and enhance the diffusion occurs at the water in powder bed ${ }^{10}$.

\section{Sintering technique}

Powder compact heated at a temperature under the melting point in which solid particles are present in controlled environment under atmospheric pressure.

\section{Polymers used in matrix tablets}

There are number of polymers which are used to formulate matrix tablets which is depends upon the physicochemical properties of the drug substance which is incorporated into matrix system and drug release profile is required ${ }^{11}$. Polymers used for matrix tablets may be classified as:

\section{Hydrogels:}

a) Poly-hydroxyethyl methacrylate (PHEMA).

b) Cross-linked polyvinyl alcohol (PVA).

c) Cross-linked polyvinylpyrrolidone (PVP).

d) Polyethylene oxide (PEO).

e) Polyacrylamide (PA).

2. Soluble polymers:

a) Polyethylene glycol (PEG).

b) Polyvinyl alcohol (PVA).

c) Polyvinylpyrrolidone (PVP).

d) Hydroxypropyl methylcellulose (HPMC).

3. Biodegradable polymers:

a) Polylactic acid (PLA).

b) Polyglycolic acid (PGA).

c) Polycaprolactone (PCL).

d) Polyanhydrides.

e) Polyorthoesters.

4. Non-biodegradable polymers:

a) Polyethylene vinyl acetate (PVA).

b) Polydimethylsiloxane (PDS).

c) Polyether urethane (PEU).

d) Polyvinyl chloride (PVC).

e) Cellulose acetate (CA).

f) Ethyl cellulose (EC).

5. Mucoadhesive polymers:

a) Polycarbophil.

b) Sodium Carboxymethylcellulose.

c) Polyacrylic acid.

d) Tragacanth.

e) Methylcellulose.

f) Pectin.

6. Natural gums:

a) Xanthan gum.

b) Guar gum.

c) Karaya gum.

d) Gum Arabic.

e) Locust bean gum 


\section{Types of matrix systems}

The matrix system can be divided into five categories depending on the types of retarding agents or polymeric materials:

1. Hydrophobic matrix system.

2. Hydrophilic matrix system.

3. Fat-wax matrix system.

4. Biodegradable matrix

5. Mineral matrix

\section{Hydrophobic matrix systems}

As the name suggests, the basic rate-controlling components of the hydrophobic matrix are water-insoluble in nature. These ingredients include waxes, glycerides, fatty acids, and polymeric materials During drug release, physical dimension of the hydrophobic matrix is necessary to maintain, so presence of insoluble ingredient is needed.

Furthermore, hydrophobic matrix systems has property to provide programmable rates of delivery which has become more important. The primary targets of controlled release system is constant rate delivery it is especially for a drug with narrow therapeutic index.

\section{Hydrophilic matrix system}

In case of hydrophilic matrix system primary rate-limiting ingredients are polymers that would swell when it came in contact with the aqueous solution and form a gel layer on the surface of the system. When the release medium (i.e. water) is thermo-dynamically compatible with a polymer, the solvent penetrates into the free spaces between macro molecular chains. Due to the stress property of the penetrated solvent, the polymer may undergo a relaxation process, so that the polymer chains become more flexible and the matrix swells. Due to this property the encapsulated drug diffuse more rapidly out of the matrix. On the other hand, it would take more time for the drug to diffuse out of the matrix, since matrix swelling lengthens the diffusion path. It has been narrowly known that swelling and diffusion are not the only factors that determine the rate of drug release ${ }^{12}$.

\section{Fat-wax matrix systems}

The absorption of drug can occurs into fat-wax granulations by spray congealing in the air, blend congealing in an aqueous media with or without the aid of a surfactant and spray-drying techniques. In the bulk congealing method, a suspension of drug and melted fat-wax is allowed to solidify and is then comminuted for sustained release granulations. The mixture of active ingredients, waxy materials and fillers also can be converted into granules by compacting with roller compactor, heating in a suitable mixture such as fluidized-bed and a steam jacketed blender or granulating with a solution of waxy material or other binders ${ }^{13}$.

\section{Biodegradable matrix systems}

Biodegradable matrix systems are composed of monomers which is linked to one another through functional groups with unstable linkage. They are degraded by enzymes generated by surrounding living cells or by non-enzymatic process into oligomers and monomers in the biological systems. Afterwords, these oligomers and monomers get metabolized or excreted. Examples are natural polymers such as proteins and polysaccharides; modified natural polymers; synthetic polymers such as aliphatic poly (esters) and poly anhydrides.

\section{Mineral matrices}

In the preparation of mineral matrices several polymers obtained from different species of seaweeds are used for example, Alginic acid, a hydrophilic carbohydrate obtained from brown seaweeds (Phaephyceae) by the use of dilute alkali. On the basis of porosity of matrix, these are classified as (a) Macro porous (b) Microporous and (c) Non-porous systems. In macro porous systems, the diffusion of drug occurs through pores of the matrix, which are of size range 0.1 to $1 \mu \mathrm{m}$. In micro porous system, the diffusion occurs essentially through pores but the pore size ranges between 50-200 $\AA$. In non-porous system no pores are found and the molecules diffuse through the network meshes. ${ }^{14}$

\section{EVALUATION OF MATRIX TABLETS}

\section{Pre-compression evaluation}

\section{Drug excipient compatibility studies}

Any incompatibility or interactions between drug and the polymer were studied through FTIR spectra and DSC. 


\section{Fourier transforms infrared spectroscopy (FTIR)}

It is conduct for configuration characterization and drug excipient compatibility. All samples dry in hot air oven at $500 \mathrm{C}$ for $2 \mathrm{hrs}$ then prepare as $\mathrm{KBr}$-disk compress under 10 ton $/ \mathrm{nm} 2$ pressure. Additional peak or lack of characteristic peak due to chemical interaction related to drug and polymer ${ }^{15}$.

\section{Differential scanning calorimetry (DSC)}

It is conduct to study the chemical interaction between active and non-active ingredients. The sample to be assayed takes in the perforated DSC aluminum pans and scan in the specified temperature range. The heating rate is maintained and nitrogen served as purged gas. The system was cooled down by liquid nitrogen. The differential thermal analyzer use for this purpose.

\section{X- Ray diffraction pattern}

XRD analysis of the drug, polymer and their physical mixture were carried out by $X$ ray diffractometry. It is run to conduct full scan with the counts being accumulated for $1 \mathrm{~s}-1$ after each step.

\section{Determination of solubility}

Solubility determine by adding an amount of compound well in excess of its saturation solubility to the solvent. Excess drug substances agitate in each buffer for few hours and then centrifuged. The solubility is checked by testing aliquot of supernatant after $24 \mathrm{hrs}$.

\section{Moisture content determination}

Moisture content determine by Infra-red drying (gravimetric method) and Karl-fischer titrations (chemical method). Thermo-gravimetric moisture balances determine moisture content in terms of the extent of weight loss that occurs as the sample is heated. Whereas in Karl Fischer titration, a reagent is added to the sample that reacts with the water and produce a nonconductive chemical ${ }^{16}$.

\section{Particle Size Analysis}

Various sieves and agitation devices are used to study sieve analysis. Each method may give different results for sieve analysis and endpoint results. Mechanical or electro-magnetic agitation method can induce vertical oscillation or a horizontal circular motion, or tapping or both. Entrainment of the particles in an air stream may also be used ${ }^{17}$.

\section{Angle of repose}

The slope of heap is checked by fixed funnel method. The height and diameters of conical pile is measured and angle of repose $(\Theta)$ is obtained by:

$\theta=\tan ^{-1}(h / r)$

$\mathrm{h}=$ height of cone

$\mathrm{r}=$ radius of conical base

\section{Porosity}

Porosity is the amount / volume of void as compared to the total amount.

Porosity $=$ Void volume / Apparent volume

\section{Density}

Both apparent \& tap denseness are measured by introducing powders into a measuring cylinder. Apparent \& tap denseness can be measured by: Apparent density = Mass / Apparent volume of occupied powder

Tapped density $=$ Mass $/$ Tapped volume of powder

\section{Compressibility (Carr's) index \& Hausner's ratio}

These percentage and ratio are determined by using following formula:

Carr's index $(\%)=[($ Tapped density - Apparent density) x 100] / Tapped density

Hausner ratio $=$ Tapped density $/$ Apparent density

\section{Post-compression evaluation}

It includes uniformity of weight, rigidness, consistency, diameters, fragility, disintegration, swelling, active drug-uniformity, and in-vitro dissolution-testing.

\section{Weight uniformity}

Weighing 20 tablets separately weigh using analytical-balance. The weight variation should be within the specified limits. Test will be failed if $>2$ tablets are not within specified values. 


\section{Dimension (thickness)}

Thickness are an important parameter to check uniformity of tablet size. Thickness and diameter are determined simultaneously ${ }^{18}$.

\section{Hardness}

Hardness is an important parameter to check physical strength of tablet. Hardness is determined by hardness tester ${ }^{18}$.

\section{Friability}

Ten tablets are weighted and placed in a friabilator then rotate for four minutes at 25-rpm. The tablets then dedusted \& reweighed. It should be preferably between 0.5 to $1.0 \%$. Formula for measuring percent friability is:

$\left[\left(\mathrm{W}_{1}-\mathrm{W}_{2}\right) \times 100\right] / \mathrm{W}_{1}$

\section{Swelling-studies}

Swelling-index is measured by putting tablets in water-filled beaker. Each tablet is weighted after different time intervals. Formula for calculating swelling-index is:

$\% S=W t-W_{o} / W o$

$(\mathrm{Wt}=$ weight after putting, and $\mathrm{Wo}=$ weight before putting)

\section{Dissolution}

Dissolution test of carried out by specified dissolution USP method at a maintained body temperature i.e. $37^{\circ} \mathrm{C}$ with specified USP Pharmacopoeial conditions. Samples are taken out at different intervals of time by using syringe, filter \& assay by HPLC or ultravioletvisible spectrophotometer method ${ }^{20}$.

\section{Conclusion:}

As a result of the preceding explanation, it is easy to deduce that sustained-release formulations aid in boosting the dose's efficiency while also enhancing the patient's compatibility. Additionally, all of these features come at a fair price. The dose form is simple to optimise and very beneficial in the case of antibiotics, where irrational usage may result in resistance.

\section{References:}

1. Yesuneh TM. 2020. Pharmaceutical solid oral dosage form analysis: Literature
Review. Op Acc J Bio Sci \& Res. 4(1), 17.

2. Khan MG. 2017. The Novel Drug Delivery System. World Journal of Pharmacy and Pharmaceutical Sciences. 6(7), 477-487.

3. Patil S, Mhaiskar A, Mundhada D. 2016. A Review on Novel Drug Delivery System: A Recent Trend. International Journal of Current Pharmaceutical \& Clinical Research. 6(2), 89-93.

4. Swaleh MM, Nisa Z, Ali SA, Khan MA, Shehnaz S. 2020. A Detailed Review on Oral Controlled Release Matrix Tablets. Int. J. Pharm. Sci. Rev. Res., 64(2), 27-38.

5. Mondal N. 2018. The Role of Matrix Tablet in Drug Delivery System. International Journal of Applied Pharmaceutics, 10, 1-6.

6. Tripathi KD, Controlled Drug Delivery System, 1-36.

7. DeRuiter J. Principles of Drug Action 2, Fall 2002. Non-Steroidal Antiinflammatory Drugs (NSAIDS), 1-26.

8. Welch E. 2017. Non-steroidal antiinflammatory drugs (NSAIDs) Part I Anaesthesia Refresher Course - 2017, University of Cape Town, 09, 01-06.

9. Bushra R, Shoaib MH, Naeem MI, Aslam N. 2013. Acelofenac: A New Effective and Safe NSAID, IJDDT, 4(1), 34-42.

10. Koirala S, Nepal P, Ghimire G, Basnet R, Rawat I, Dahal A, Pandey J, Baral KP. 2021. Formulation and evaluation of mucoadhesive buccal tablets of Aceclofenac. Heliyon, 7, 1-10.

11. Bhalsing MD, Shekade SV. 2020. Formulation And In Vitro Evaluation of Colon Targeted Drug Delivery of Aceclofenac. Pharmaceutical Resonance, 2(2), 34-40.

12. Prasanna Kumar PSS, Ravi Kumar A, Srinivas N, Durga Prasad P, Bhargavi S, Vidya Sri T, Ramya V, Bala Sowjanya V. 2019. Design, Formulation and In-Vitro Evaluation of Aceclofenac Fast Disintegrating Tablets Using Natural Polymers. International Journal of 
Pharmaceuticals and Health care Research, 07(1), 23-34.

13. Khatik T, Moravkar K, Suryawanshi D, Shinde U, Amin P. 2018. Development of sustained release Aceclofenac lipid matrix tablet using continuous melt granulation technique. American Journal of PharmTech Research, 8(3), 233-248.

14. Wadher KJ, Kalambe P, Umekar MJ. 2017. Formulation and Evaluation of Colon Targeted Aceclofenac Sodium Sustained Release Tablets. International Journal of MediPharm Research, 03(1), 229-236.

15. Kang WH, Nguyen HV, Park C, Choi YW, Lee BJ. 2016. Modulation of microenvironmental $\mathrm{pH}$ for dual release and reduced in vivo gastrointestinal bleeding of aceclofenac using hydroxypropyl methylcellulose-based Bilayered matrix tablet. European Journal of Pharmaceutical Sciences, 5(3), 1-38.

16. Culcu OG, Arisan I. 2015. Formulation Development, in vitro Evaluation and
Stability Study of Aceclofenac Tablet, J. Chem. Chem. Eng., 9, 101-106.

17. Gandhi A, Harikumar SL. 2015. Formulation and Evaluation of Aceclofenac Matrix tablets using hydrophobic waxes for sustained release. Indian Journal of Drugs, 3(3), 49-56.

18. Jana $\mathrm{S}$, Sen KK, Basu SK. 2014. In vitro aceclofenac release from IPN matrix tablets composed of chitosan-tamarind seed polysaccharide. International Journal of Biological Macromolecules, 65, 241245.

19. Gupta CR, Kishore GK, Ratna JV. 2013. Development and evaluation of aceclofenac matrix tablets using polyethylene oxides as sustained release polymers. Journal of Pharmacy Research, 6, 249-254.

20. Dey S, Mahanti B, Khila S, Mazumder B, Gupta SD. 2012. Formulation development and optimization of bilayer tablets of Aceclofenac. Expert Opin. Drug Deliv., 9(9), 1041-1050. 